\title{
EXPERIENCE IN THE TREATMENT OF WAR BURNS
}

BY

\author{
SOL. M. COHEN, M.A., F.R.C.S. \\ Surgeon, Sector 10, E.M.S
}

The flash of the exploding bomb and the fires started by incendiary bombs make burns one of the most important of air raid casualty injuries. Afte: the evacuation from Dunkirk a large number of cases of such burns were transported to the hospital to which I am attached. Many lessons were gathered, but one lesson stood out clearly from it all-the necessity for and the value of a prepared routine of treatment.

With numerous other casualties requiring treatment much of the after-care of these cases must be left to the nursing staff, and it is essential, first of all, that instructions to them be clear and definite. In the majority of cases both hands are burnt and the eyes are closed. The demands on the nursing staff are consequently heavy; therefore it is a mistake to fill a ward with burn cases only. On the other hand, the cases should not be distributed to more than three or four wards. It is then easier for the surgeon and medical officer, the amount of stained linen is much reduced, and a few sisters are enabled to become experts in treating burn cases. In the midst of patients recovering from other injuries, who talk to them and help them with their comforts, convalescence too is brightened.

In view of the damage to the skin, there has been a tendency to feel that skin specialists should regard these cases as coming within their domain. "Burn clinics" under their care have even been formed. But these cases require a great experience of sepsis, of deformities, of ill patients, of blood transfusions, and a knowledge of skin grafting. The responsibility for them should be in the hands of a surgeon, who, though he will not be able to attend personally to all, must yet guide the treatment. The aid of other specialists must, however, be freely invoked. Thus one of the ophthalmologists of this sector saw the eyes in every burn case, and a standard routine method of treatment was mapped out. A dermatologist, too, was invited to see the cases, and he was able to give much help in treatment. It is often the custom to relegate these cases to the most junior house-surgeons. The swollen face and eyes, the mask of the tan, make it not easy to judge the clinical condition of a case of burnsand here the value of the physician cannot be overstressed. At this hospital these cases came under the care of the physicians. They are able to guide as to the general condition of the patient and give much assistance in the problems of septicaemia, nephritis, and chest complications-though these were but occasional in this series. I would urge that in wartime physicians be responsible for cases of burns-under the guidance of the surgeon and with his co-operation.

\section{General Treatment}

The soldier is of course a "fit" patient and he will as a rule withstand more than the civilian. Of the patients admitted from Dunkirk some had been almost five days and five nights without sleep. After constant bombing and machine-gunning from the air, shell fire on the sands, and immersion in the sea they were exhausted. Sleep and rest were as important as operation. With prolonged air attacks at night these conditions can well be repeated in this country. As a routine, therefore, morphine was immediately given in large doses-and it should be stressed that a third of a grain is usually required. Some of the men admitted had been given fat too small doses. Following morphine, 1/3 grain of omnopon was "boarded" as a routine in every case; this was to be given if the patient was in pain-and the nursing staff had it impressed upon them that it was not a feat or a credit to have withheld this. Many of the men had been lying on the sands at Dunkirk after their burns, and as they all did so well it has been suggested that the immersion in the sea-water had probably been the best cleanser. Be that as it may, a large number had not been immersed, and they too did well. With such conditions it can well be appreciated that some of the men were very ill on admission-and it is difficult to judge how ill a burn case is, especially by the light of a small torch. Blood-pressure readings are suggested as a routine, but nearly all these patients have their forearms and hands burnt and cedematous, and it is agony for them to have the blood pressure taken. Frequent haemoglobin readings are recommended; but with a large number of casualties, and darkened wards, it is impossible, however desirable, to carry this out. Clinical signs alone had to serve as a guide to the condition of the patient, and of these the pulse was always the most reliable. The ill patient lies still, moving not a limb--cold in body and, despite the morphine, groaning in a weak voice. In two cases 500 c.cm. of plasma - as recommended by Wilson (1940)-was given; they recovered, but the number is too small to prove anything. For those patients who were cold the hot electric cradles usually recommended were not available, but hot-water bottles in large numbers sufficed. The old-fashioned remedy of $6 \mathrm{oz}$. of hot strong coffee per rectum proved most satisfactory in warming up these patients. With burnt faces and lips any hot drinks were resented

\section{Local Treatment}

As an immediate application tannic acid, eitther in solution or as a jelly, is unquestionably the method of choice. Several men had been burnt in tanks three to five days before admission. All tanks carry first-aid tannic jelly outfits-and hands filled with grease and oil had daubed the jelly all over. These cases did well: not only was the area coagulated but their general condition was so satisfactory because any further loss of serum had been sealed off by the tan. This should be the routine treatment. Many of the cases transported here had had picric acid or acriflavine and paraffin applied at the first-aid stations.

Every patient was cleaned in the theatre as soon as his condition permitted; and, with fluids by mouth and rectum only, and plasma in two cases, this was possible. Many surgeons are reluctant to clean burns admitted late ; but in our experience the general condition in no way deteriorated with this cleaning, nor did it precipitate seveı e sepsis or septicaemia. All these patients had received their burns at least twenty-four hours before admission, the majority three to four days previously. No case was "too late" for full treatment in the theatre. The burns were chiefly confined to the face and scalp, the forearms and hands; and in the more ill patients the skin of the chest or abdomen was burnt. In some the entire skin over these regions was charred, but in the majority the total thickness of skin was not involved, and they may therefore be classed as the "superficial" or second and third degree burns.

Anaesthesia.-Nearly all these men had burnt faces. Intravenous anaesthetics such as evipan and pentothal spared the patient the unpleasantness and difficulties of induction. A vein in the arm or the leg was always available. This type of anaesthesia has been condemned as too " depressing" for such cases. In no instance, 
however, was there any trouble, and it was made a rule that the maximum dose of evipan was to be $10 \mathrm{c.cm}$. (1 gramme). It was necessary to continue with gas and oxygen in most cases, and the mask was applied over several layers of sterile gauze. The intratracheal tube is of course ideal ; but its use calls for some dexterity and experience, and the expert anaesthetist was usually fully occupied with the severer casualties. With the large number of burns to be dealt with, all available operating tables had to be utilized, and the anaesthetics had therefore to be divided among the remaining staff. The expert anaesthetist may well serve best by supervising a number of cases.

The Cleaning.-With arms and face all burnt, the work may be expedited by a nurse or a second doctor cleaning at the same time as the surgeon and under his supervision. Much has been written on the need for gentleness in these cases, and it has been recommended that only saline be used. Nearly all these patients, however, have grimy oily hands, on which the saline makes no impression. Weak ether soap and water was utilized in all these cases. The soap is smooth, and washing-not scrubbing-will merely remove the debris and add nothing to the damage. Many of these patients were still covered with the fuel oil of the sunken ships: with ether soap this readily came off. The region surrounding the burn is the important area, for from that will come the infection. In all face and forehead burns the hair was therefore shaved well back under the anaesthetic. No scrubbing-brush was used and the surrounding skin was washed and prepared as if for operation. While the burnt area itself is being cleaned it is well to ask the assistant to picture the battered frail underlying cells that guard a surface, apparently smooth, but jagged and irregular as the "fjords of the coastline of Norway"-as Mr. Ogilvie has so picturesquely described it. The debris, the dead skin, and all the overlying blisters are removed, and it is common for dead dirty nails to come off in the process. Attempts are often made unnecessarily to leave these, and they form a potent source of infection.

The Silver-nitrate-Tannic-acid Method.-With a large number of burns the slower methods of coagulation cannot be applied. To spray every fifteen minutes or to be continually applying more solution disturbs the patient at the time when he needs most rest, and when the nursing staff will already have been worked to the limit. An immediate tan is undoubtedly a great advantage, and this method was used throughout and proved most satisfactory. After cleaning with ether soap the surface was washed with saline, and the surrounding area, spirited, rapidly dried. The solutions $-5 \%$ tannic acid in water and $10 \%$ silver nitrate-are kept separate and are dabbed on to the cleaned areas by separate gauze swabs. The black tan which immediately forms rapidly dries. The silver nitrate solution keeps, but the tannic solutions are preferably freshly prepared. On the face a little petroleum jelly is first applied to the eyelids, and if two pieces of wool are held firmly over them there is no danger of the solution running into the eyes. The crust is not the thick crust of stronger tannic-acid solutions, and therefore moulds itself more readily to the changing contours in the regions of the joints. It has been urged that there is some danger of a tannic-acid crust causing gangrene of the fingers-and for this reason methyl-violet has apparently found favour. In the only case in our series in which the tips of some fingers sloughed the gangrene was primarily due to the burn and was anticipated at the time of the primary treatment. The scorched dark brown epidermis is often not recognized as indicating severe deep damage. A series of patients admitted at the same time who had been treated with methyl-violet had a very much more prolonged and unsatisfactory convalescence.

Splintage.-It is characteristic of burns that a marked local oedema develops within a few hours. Not only does this increase the discomfort of the patient but its persistence will lead to "glueing," especially of the fingerjoints, and convalescence will be delayed. As a routine the burnt forearm and hands were therefore placed on broad straight padded Cramer wire splints, with a pad of wool for the palm and pieces of gauze between the fingers. They were then wrapped in gauze and bandaged to the splint. In bed the raising of the splinted arms on sloping pillows greatly assisted in the rapid reduction and drainage of the oedema. This maintains the wound at rest ; it is so often forgotten that burns are wounds and must be treated as such. The patient's hands or face must not be allowed to roll in the blankets or sheets, for that is a most potent cause of sepsis in these cases. The face and neck should not be bandaged at all, but sterile towels should be placed under the head and the chin. It must be stressed that the splints must be lightly applied, and that their purpose is in no way connected with the prevention of contractures.

Brilliant-green.-The burnt areas are usually infected from the adjacent skin, and the streptococcus is the commonest type of invader. Brilliant-green is particularly effective in treatment of infection, and in our cases the edges of the tan were painted daily. More frequent painting causes no damage when there is a tendency to moistness. We used $1 \%$ brilliant-green in $30 \%$ alcohol as recommended by Henry. This is of special importance in burns of the scalp, forehead, and neck. In all the cases except two it was possible to keep the scabs in these regions absolutely dry.

The Eyes:-The eyes require much care. With small pieces of sterile wool dipped in $1 \%$ solution of sodium bicarbonate the excessive secretion is wiped away several times a day and a few drops of sterile liquid paraffin (parolein) inserted. The eyes are kept unbandaged, and if there is any marked redness the nursing staff is instructed to wash them out. It is amazing how the eyes escaped injury in all these explosive burns. Only one patient developed a small corneal ulcer.

\section{Chemotherapy : Diet}

Chemotherapy was employed as a routine, with a standard dosage. An initial dose of three tablets of sulphanilamide was followed by two tablets every four hours for forty-eight hours and then one tablet four-hourly for another forty-eight hours-a total of 19.5 grammes. By this method inadvertent overdosage is avoided. Sulphapyridine was not used in these cases because it is our experience that despite glucose and sodium bicarbonate there is a tendency to nausea or vomiting. As it is important for these patients to take ample fluids, even mere nausea is obviously a disadvantage. No dose of sulphanilamide is omitted even at night-and this has to be stressed, for some of the patients under morphine may well sleep twelve hours on end and thus miss three doses. With this sulphanilamide dosage there were no complications.

Only one patient developed a leucopenia: his white cell count fell to 2,250 (polymorphs $22 \%$, lymphocytes $70 \%$ ). In his case there were also an extensive laceration of the scalp and bilateral fractured calcanei. Because of coninued swinging pyrexia, without obvious pocketing of pus, his house-physician placed him on a course of sulphapyridine. The course began one week after the sulphanilamide therapy ended, and he was given 22 grammes in a period of seven days. With cessation of the drug and the use of pentnucleotides the white cell 
count rose within forty-eight hours to 4,450 (polymorphs $40 \%$, lymphocytes $52 \%$. This patient is also of interest as he was the only one in this series who passed blood and albumin in the urine-in the sixth week. There was no pain, no oedema, no casts, no hypertension. Haematuria has been described after sulphapyridine therapy, but the administration of that drug had ceased three weeks previously.

With the large number of cases now having chemotherapy at the same time a standard dosage in each hospital is most desirable.

Diet is undoubtedly important. Much protein has been lost via the serum, and a higher protein diet is therefore advisable. Milk supplies protein in the most abundant manner, and the patients are therefore encouraged to drink large quantities. Vitamins play an important part in the repair of tissues, and although synthetic vitamins may be used the natural food vehicle is always the best. Each patient was therefore ordered a full fruit ration, and, as ample oranges were available, four fresh oranges and two lemons daily in the form of drinks, extra fresh tomato juice, and their full share of butter.

The above instructions are best summarized by giving each burns ward a slip worded as follows:

\section{BURNS CASES}

1. Hourly pulse for 24 hours.

2. Fluid chart. Urinary output.

3. Push the fluids, especially milk. The patient requires a high protein diet. Include four oranges and two lemons in his daily drink.

4. Remember, burns are open wounds and require the same 4. Remember, burns are open wounds and require the same
asepsis. The discharge of pus is not an indication of tan. failure -it is inevitable with granulations. Frequent dressings serve no purpose and are harmful.

5. Keep the affected limbs elevated. Encourage the active movements of all other joints.

6. Treat the Eyes.-Excess pus is wiped away several times a day with sterile cotton-wool in $1 \%$ sodium bicarbonate solution. A few drops of parolein are then inserted. The eyes are not washed out unless they are very red.

not washed out unless they are very red. 7. Sulphanilamide Therapy.-Tabs. iii stat. Then 2 tablets
4-hourly for 48 hours; then 1 tablet 4-hourly for another 48 4-hourly for 48 hours ; then 1 tablet 4-hourly for another 48
hours. No dose is to be omitted even at night. This dosage is not to be further continued without the medical officer"s instructions.

8. A spirit of encouragement, and assurance, will do much to ease the convalescence.

\section{Later Treatment}

Dressings. - The limbs are maintained splinted for seven days, when the scabs will have begun to lift and it will be essential to start movements of the fingers. Healthy joints, we know, as Watson Jones especially has stressed, may be immobilized for a considerable time and a full range of joint function expected. But with fingers, unless early movement is re-started the return of full function is much delayed. The explanation can be found in the fact that clothing all other joints there are muscles, which despite immobilization continue to massage, and maintain the circulation in, the lymphatics. In the fingers there are no muscles, and unless tendon movements are retained the lymph stagnates and becomes organized round the joints. Within a week, if healing has been maintained, the hands and fingers therefore come out of their splints, a sterile dressing is lightly bandaged on over the tan, and active movements are started. There is no danger of the tan being damaged by active movements, for hands that can bend iron will not bend a straw if this causes pain.

Deeper Burns.-The lifting of the tan need not bring disappointment. If the burn has been deeper granulation tissue is inevitable and there will be pus under the tan. But this is no indication for its removal. No further dressing was done in these cases until the fourteenth day, when the tan, if already lifted, was removed. Gauze with vaseline was then applied and left for a further fortnight. Several cases were re-tanned, but if the burn is deep this again lifts, and no great advantage was obtained. Small areas between the fingers and at the corners of the ears, the tip of the nose, or the dorsum of the hand are occasionally unhealed when the crusts lift. Every sister has a pet ointment, but in our experience none of many proprietary preparations showed any advantage over the simple petroleum jelly dressing. It is important to impress upon the patient that dressings are being deliberately avoided, otherwise he is apt to feel neglected.

Contractures.-For deep burns it cannot be too strongly emphasized that to prevent scarring contractures skingrafting at the earliest possible moment after the sloughs have separated is advisable. Tight splints will not prevent contractures; often they favour it. As the damaged tissue is maintained on the stretch it lays down more and more scar tissue, which will always contract. A skin graft alone will prevent formation of scar tissue. However severe the burns no cock-up splints or correcting plaster slabs were employed. Nothing is to be gained by the daily dressing of these burns with eusol and suchlike antiseptics. Nature will do her own separation far more proficiently and adequately. Only a simple dressing of petroleum jelly was used.

Plaster-of-Paris.-The same principles of healing apply to burns as to other wounds, and the importance of immobilization of the affected part is recognized by all. The use of plaster for the limbs therefore commends itself; but the extensive oedema so characteristic of burns which develops within a few hours obviously limits its employment. In the Dunkirk casualties the oedema was already established, and the few patients upon whom plaster was tried were very uncomfortable. In two associated forearm fractures a close-fitting plaster was applied over petroleum jelly, but in forty-eight hours the oedema had subsided and the plasters were loose shells.

In the later treatment of the deeper severe burn cases, however, plaster proved of great value. At the end of a fortnight the oedema had subsided, and the use of plaster then began. In this series it was employed in seven extensive burns of forearm and hand, and it had been effective in lower-limb cases too. Over a vaseline dressing and one layer of sterile plaster wool-the vulnerability of burns to any pressure is well known-the forearms and hands were encased in plaster. The improvement was immediate: the patients all felt comfortable, and by the simple addition of a pen encased in or strapped to the plaster they. were able to turn over the pages of their books. The plasters were changed at fortnightly intervals. At the end of six weeks two of the limbs were found to be healed. Four others were skin-grafted, and it was found that the Thiersch grafts "took" even if applied immediately after removal of the pus-soaked plaster. No preliminary "cleaning up" for several days with saline compresses, as is often done, was necessary. In one case, where the initial burn was so severe as to have destroyed the tips of the fingers, it was already possible to Thierschgraft the greater part of the hands and forearm.

\section{Results}

Seventy cases of burns were admitted. Of these 37 were treated in the theatre by the silver-nitrate-tannic-acid method; the rest had been cleaned up and treated before admission by either methyl-violet or tannic-acid solutions. There were no deaths in the silver-nitrate-tannic-acid series. There were, however, two deaths in the otherone patient died immediately on admission and one died four days later. The former case had been cleaned, treated with tannic acid, and immediately transferred to this hospital. In our experience, with the use of chemotherapy the later cleaning of such a burn-that is, after transportation-does not add to the risk. The other case 
had been partially cleaned and treated with methyl-violet, and the patient died four days after admission.

The average healing time with the silver-nitrate-tannicacid method was three and a half weeks, and the aesthetic appearance of such scars was particularly gratifying. At the seventh week the recently grafted cases alone had not yet regained full movements of the fingers.

\section{Summary}

The principles of the treatment of burns, with special reference to the Dunkirk cases, are discussed.

The importance of a prepared scheme of treatment, especially in war, is stressed.

The silver-nitrate-tannic-acid method is strongly recommended.

The use of plaster for the later treatment of deep burns is advocated.

Wilson, W. C. (1940). E.M.S. Memorandum (Gen. 314).

\section{ABDOMINAL ACTINOMYCOSIS TREATED WITH SULPHAPYRIDINE}

BY

\section{W. H. OGILVIE, M.Ch., F.R.C.S.}

Surgeon to Guy's Hospital

The poor prognosis in abdominal actinomycosis is sufficient justification for reporting the recovery of a case with diffuse infection after treatment with sulphapyridine.

\section{Clinical History of Case}

The patient, a girl aged 22 in the A.T.S., was curetted for dysmenorrhoea on November 13, 1939. She recovered without incident from this operation, but on November 17 she complained of abdominal pain and her temperature rose to $104^{\circ}$. On November 20 a laparotomy was performed through a small right paramedian incision and a drainage tube was inserted. On November 25 pus appeared for the first time, and thereafter continued to flow round the tube in increasing quantities. She lost weight and strength and complained of constant abdominal pain. On two occasions she was transfused.

I saw her first on December 19, a pale emaciated girl dreading every contact. Light creamy pus was pouring round the tube in the abdominal incision; it did not appear to be coming from the abdominal cavity, but from pockets in the abdominal wall that extended over the whole lower part and into both labia, upwards round the umbilicus, and over the epigastrium as far as the costal margin and outwards into the right flank. So far as could be felt by palpation through this infected abdominal wall there were no masses in the abdomen, and respiratory movements were reasonably good. Rectal examination suggested a plastic peritonitis binding the pelvic viscera together but did not disclose any lump or localized abscess. It was clear that the incision was merely a vent through which the overflow from a large undrained reservoir of pus was escaping in quite inadequate quantities. The wide extension of this pus in the subcutaneous planes of the abdominal wall, and its appearance, suggested that the case was an example of the undermining ulcer described by Frank Meleney (1936), which is due to a micro-anaerobic streptococcus and responds readily to free drainage and treatment with zinc peroxide. I asked that the girl might be transferred to London, where I had a supply of active zinc peroxide from the Dupont Company, kindly presented to me by Meleney when I was in New York. She was unfit to stand the journey of sixty miles, however, so I suggested that she should be transfused to bring her haemoglobin up to at least $70 \%$ and be sent in an ambulance as soon as her condition would admit it.

She arrived on December 29 very much as 1 had seen her, improved by the transfusion but debilitated by the reaction which had followed it and exhausted by the journey. I operated on January 1, 1940, laying open all the sinuses and packing them with vaseline gauze. The lower collection communicated with the extraperitoneal tissues of the petvis through openings in each inguinal canal. The prolongation upwards led to a large cavity between the superfrcial fascia and the rectus sheath in the epigastrium; from this a track led through the abdominal wall under the right costal margin to the subdiaphragmatic space, apparently between the liver and the diaphragm, and a second track back into the perinephric tissues in the right loin. After this operation she was very collapsed, her haemoglobin dropping from $70 \%$ to $49 \%$, and she was again transfused.

Scrapings from the granulation tissue showed polymorphonuclear infiltration and Gram-positive cocci. Stained films of the pus revealed a streptothrix, a Gram-positive coccus, and a. Gram-negative bacillus. Cultures yielded Streptothrix actinomyces. She was given 180 grains of potassium iodide by mouth daily, which she tolerated well, and 90 grains of Blaud's pill daily with injections of anahaemin $2 \mathrm{c} . \mathrm{cm}$. weekly. At first the free drainage of her sinuses produced a striking improvement. Weekly blood counts showed a steady rise in haemoglobin and red cells, with a drop in the white cell count from the first reading of 56,000 to about 20,000 . The temperature remained at or just above normal. On the other hand, after an initial improvement from her cadaveric look, her appearance remained sickly, her pulse stayed at about 100, the discharge from the wound was as profuse as ever, and the granulations began to look less healthy. By the seventh week it appeared that in spite of maximum doses of potassium iodide she had ceased to improve and was losing ground, or at best was stabilized at a level from which recovery seemed unlikely.

Treatment with Sulphapyridine.-At this point Colonel Summerhayes of Newhaven, the medical officer to her unit, called my attention to the report by Miller and Fell (1939)

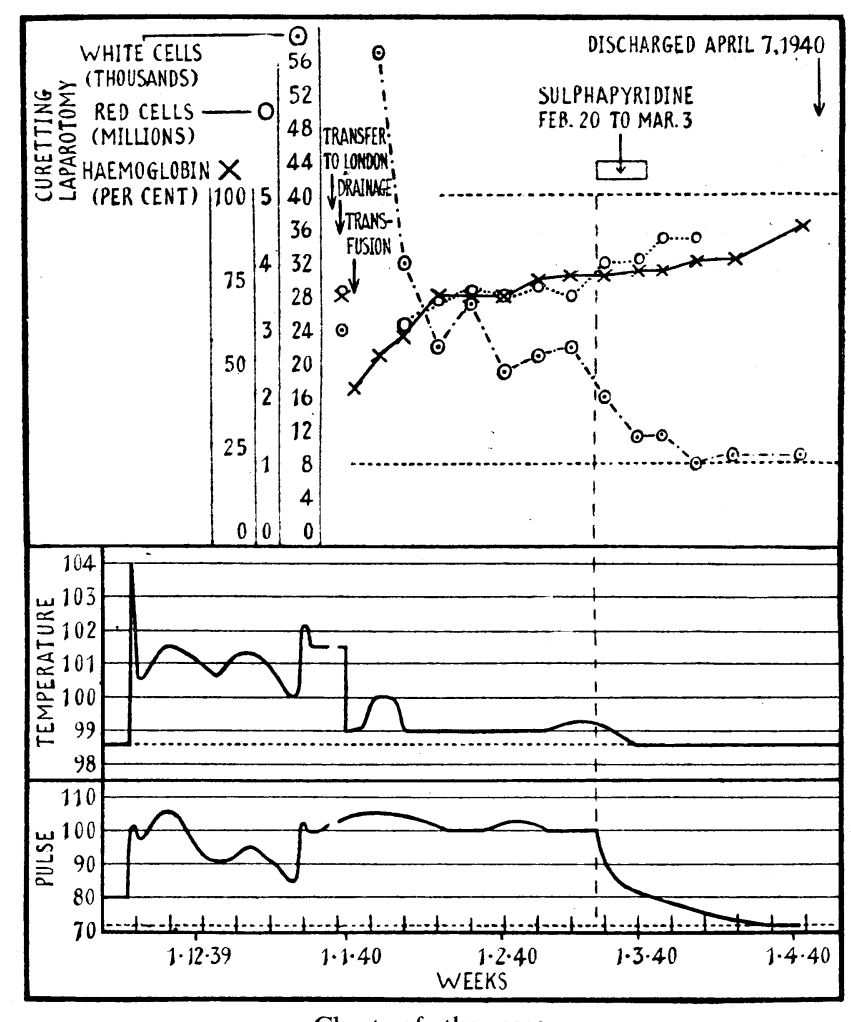

Chart of the case

of a case of abdominal actinomycosis treated with sulphanilamide. After consulting this paper and learning from it of Walker's (1938) successful case I felt that a trial of sulphapyridine was well worth while. The potassium iodide was discontinued on February 22, and on the 23rd the administration of sulphapyridine (M \& B 693) was started. At first two tablets were given four-hourly, but since this led to 